\title{
Numerical Simulation of Cavitating Water Jet by a Compressible Mixture Flow Method*
}

\author{
Guoyi PENG**, Seiji SHIMIZU** and Shigeo FUJIKAWA*** \\ ** Department of Mechanical Engineering, College of Engineering, Nihon University \\ Koriyama, Fukushima, 963-8642, Japan \\ peng@mech.ce.nihon-u.ac.jp \\ *** Graduate School of Engineering, Hokkaido University \\ Sapporo, Hokkaido, 060-8628, Japan
}

\begin{abstract}
Concerned on the numerical simulation of high-speed water jet accompanied with intensive cavitation, a practical compressible mixture flow method is developed by coupling a simplified estimation of bubble cavitation and a flexible compressible flow computation procedure. Two-phase fluid media of cavitating flow are treated as a mixture of liquid and bubbles, and the mean flow of the mixture is computed by applying Reynolds Averaged Navier-Stokes equations for compressible fluids considering the effect of bubble expansion $\mathrm{a} / \mathrm{o}$ contraction. The intensity of cavitation is evaluated by gas volume fraction which is governed by the compressibility of bubble-liquid mixture at the current status of mean flow field.

High-speed submerged water jets issuing from a convergent-divergent nozzle are treated under different cavitation conditions. The results demonstrate that pressure decreases in the convergent section and cavitation occurs initially in the low-pressure region behind the throat near the wall. The gas volume fraction of cavitation bubbles reaches to 0.5 locally when the cavitation number is decreased to 0.1 , and cavitation bubbles generated in the shear layer near the throat flow downstream along the jet periphery. Under the effect of cavitation bubbles the mass discharge coefficient of water jet decreases obviously compared to the case of no-cavitating one although the maximum velocity at the throat increases.
\end{abstract}

Key words: Submerged Water Jet, Cavitation, Turbulent Flow, Two-Phase Flow, Computational Fluid Dynamics

\section{Introduction}

High-speed water jets have been applied in many fields of industries such as cleaning of complicated mechanical products, cutting of solid materials, etc. As a new technology utilizing cavitation phenomena effectively, submerged water jets have received much attention $^{(1),(2)}$. However, the structure of high-speed cavitating jet and the behavior of unsteady cavitation bubbles are still unclear because of difficulty for observing the interior of cavitating flows ${ }^{(3)}$. For the purpose of performance prediction and efficient design of water jet devices much attention has been attracted to the numerical simulation of cavitating flow. Modeling of cavitation has been studied for many years from different view points ${ }^{(4)-(6)}$ and methods developed can be mainly classified into two different categories of two-fluid one and two-phase mixture flow one. The two-fluid method treats cavitation phenomena by computing liquid and gas flows separately, and the computed flow fields strongly depend upon physical models used for evaluating the interaction between the liquid and bubbles ${ }^{(7)-(9)}$. For lack of a general method evaluating the interaction of liquid and bubbles an extra effort 
is required in applying the method to high-speed cavitating jets.

On the other hand, cavitation usually takes place in low-pressure regions of relative high velocity and the size of cavitation bubbles is very small compared to its flow field. Cavitation bubbles and working liquid may be sufficiently mixed and their relative motion in a small local area is often insignificant ${ }^{(10)}$. Thus, the so-called two-phase mixture flow method treats the flows of liquid and cavitation bubbles together by neglecting the velocity slip between the liquid and bubbles. For its convenience the method is often used under certain simplifications of flow phenomena by combined with some cavitation models ${ }^{(10)-(12)}$. Generally speaking, the computation of two-phase mixture flow method is cheaper than that of two-fluid method since there is no need to treat the motion of a mass of bubbles separately. But the treatment of sharp density change between cavitation bubbly region and surrounding liquid region becomes a difficulty. Thus, Peng et al. advanced a flexible pressure-based computation procedure by applying the CIP-CUP method for dealing with both the compressible and incompressible features coexisted in cavitating flows ${ }^{(13)}$. To consider the unsteady bubble dynamics property of cavitation phenomena a compressible mixture flow bubble cavitation model was proposed by coupling the Two-fluid Three-pressure bubble cavitation model ${ }^{(7)}$ into the above mixture flow computation ${ }^{(14)}$. However, it is too time-expending to be applied to engineering cavitation flows since the time scale of bubble oscillation is much smaller than that of mean flow field variation.

Focused on the numerical simulation of high-speed water jet accompanied with intensive cavitation this paper presents a practical compressible mixture flow method by adopting a simplified estimation of bubble cavitation for the computation of high Reynolds number turbulent cavitating flow. Two-phase fluid media of cavitating flow are treated as a mixture of liquid and spherical gas bubbles whose interior pressure is supposed to vary almost similar to the surrounding liquid pressure. The mean flow of two-phase mixture is computed by applying Navier-Stokes equations, and the flow turbulence is modeled with the RNG $k-\varepsilon$ model by introducing a modification on the eddy viscosity of cavitating flow. The intensity of cavitation in a local field is evaluated by the volume fraction of gas phase, which is governed by the compressibility of bubble-liquid mixture at the current status of local mean flow field. The method is applied to treat high-speed submerged water jets and its validity is confirmed by comparison with experiment data. The structure of cavitating and no-cavitating jets issuing from a divergent-convergent nozzle is investigated.

\section{Compressible Mixture Flow Cavitation Model}

\subsection{Compressibility of Bubble-liquid Mixture}

The fluid media of cavitating flow are taken as a two-phase mixture of working liquid and cavitation bubbles. The gas phase is supposed to disperse in the liquid phase and its volume faction is denoted as $\alpha_{G}$. The liquid volume fraction is written to be $\alpha_{L}$. Then, the density of two-phase mixture can be defined as follows by volume averaging.

$$
\rho_{M}=\rho_{L} \alpha_{L}+\rho_{G} \alpha_{G}
$$

where $\rho$ denotes fluid density and the subscripts $L, G$ and $M$ do the liquid phase, the gas phase and the two-phase mixture, respectively. Concerning the compressibility of the two-phase mixture, the variation of mixture density can be written as follows by taking the differential of above equation.

$$
\frac{d \rho_{M}}{d t}=\alpha_{G} \frac{d \rho_{G}}{d t}+\alpha_{L} \frac{d \rho_{L}}{d t}+\left(\rho_{G}-\rho_{L}\right) \frac{d \alpha_{G}}{d t}
$$

Considering the continuity of mixture flow, we arrange it to the following form.

$$
\frac{1}{\rho_{M}} \frac{d \rho_{M}}{d t}=\frac{\alpha_{G}}{\rho_{G}} \frac{d \rho_{G}}{d t}+\frac{\alpha_{L}}{\rho_{L}} \frac{d \rho_{L}}{d t}
$$


According to the definition of compressibility the above equation is then written as follows.

$$
\frac{1}{\rho_{M}} \frac{d \rho_{M}}{d t}=\frac{\alpha_{G}}{\rho_{G} c_{G}^{2}} \frac{d p_{G}}{d t}+\frac{\alpha_{L}}{\rho_{L} c_{L}^{2}} \frac{d p_{L}}{d t}
$$

where $p$ and $c$ denote pressure and sonic speed in a given fluid respectively. Both the liquid and the gas included in bubbles are supposed to work exponentially and their state equations are given as follows.

$$
\frac{p_{L}+B}{p_{0}+B}=\left(\frac{\rho_{L}}{\rho_{L 0}}\right)^{n_{L}}, \quad \frac{p_{G}}{p_{G 0}}=\left(\frac{\rho_{G}}{\rho_{G 0}}\right)^{n_{G}}
$$

where $n_{L}$ and $n_{G}$ denote specific heat ratio of the liquid and the gas. The subscript 0 denotes a reference state which is taken to be the atmospheric one. $B$ is a constant given to be $3.049 \times 10^{8} \mathrm{~Pa}$. Then, sonic speeds in the liquid and gas media are written as the function of working pressure.

$$
\rho c_{L}^{2}=n_{L}\left(p_{L}+B\right), \quad \rho c_{G}^{2}=n_{G} p_{G}
$$

According to above we understand that the compressibility of the two-phase mixture depends upon the volume fraction of gas phase as well as the variation of gas pressure, which is determined by bubble size. Thus, the compressibility of a bubble-liquid mixture needs be evaluated based on a careful consideration of bubble dynamics, but it is very time consuming. In order to work out a practical method for engineering applications a simplification on the gas pressure that $d p_{G} / d t \approx d p_{L} / d t$ is adopted and then Eq. (4) is arranged to the following form.

$$
\frac{1}{\rho_{M}} \frac{d \rho_{M}}{d t}=\frac{1}{\rho_{M} c_{M}^{2}} \frac{d p_{L}}{d t}
$$

where the average sonic speed in the mixture is defined by the following equation.

$$
\frac{1}{\rho_{M} c_{M}^{2}} \cong \frac{\alpha_{G}}{\rho_{G} c_{G}^{2}}+\frac{\alpha_{L}}{\rho_{L} c_{L}^{2}}
$$

\subsection{Governing Equations for Turbulent Cavitating Flow}

The mean flow of two-phase mixture consisting of liquid and bubbles is concerned by neglecting their relative velocity slip. Considering the variation of mixture density caused by cavitation Reynolds Averaged Navier-Stokes equations for compressible fluid are adopted as main flow governing equations. The variation of temperature caused by cavitation is thought to be very small in the whole flow field and thus the conservation equation of energy is omitted. Conservation equations of mass and momentum employed are given as follows in vector form. For convenience the subscript $M$ denoting the fluid mixture is omitted hereafter.

$$
\begin{aligned}
& \frac{\partial \rho}{\partial t}+\boldsymbol{u} \cdot \nabla \rho=-\rho \nabla \cdot \boldsymbol{u} \\
& \rho \frac{\partial \boldsymbol{u}}{\partial t}+\rho(\boldsymbol{u} \cdot \nabla) \boldsymbol{u}=-\nabla p+\nabla \cdot \boldsymbol{\tau}+\boldsymbol{g}
\end{aligned}
$$

where $\boldsymbol{u}$ denotes the mean velocity vector and $\boldsymbol{g}$ does the gravity. $\tau$ denotes the stress tensor and its components are given as follows.

$$
\tau_{i j}=2 \mu S_{i j}-\frac{2}{3} \mu(\nabla \cdot \boldsymbol{u}) \delta_{i j}-\overline{\rho u_{i}^{\prime} u_{j}^{\prime}}
$$

in which $i$ and $j$ denote respectively three components of the coordinates. $\delta_{i j}$ denotes the Kronecker delta and $S_{i j}$ does the strain tensor. $-\overline{\rho u_{i}^{\prime} u_{j}^{\prime}}$ does the Reynolds stress which is related to mean velocity filed via a turbulence model. $\mu$ does the mean viscosity of the two-phase mixture and it is estimated by the following formula given by Beattie et al. ${ }^{(15)}$. 


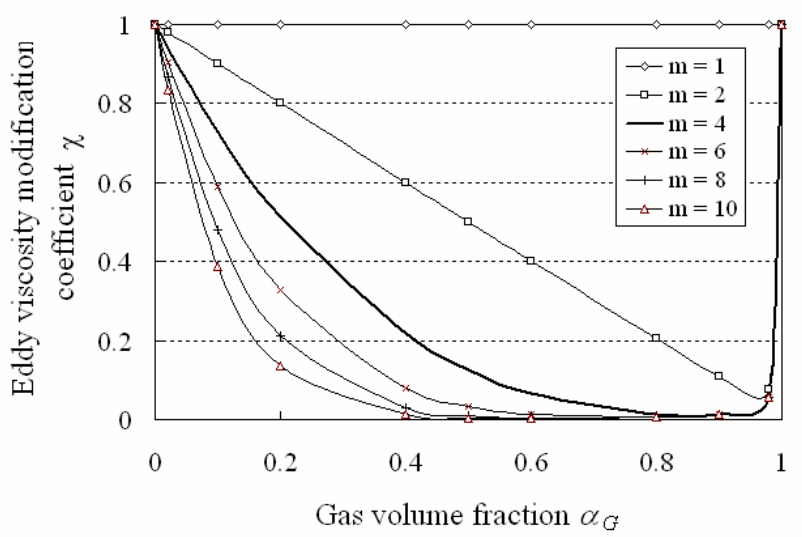

Fig. 1 Eddy viscosity modification coefficient dependent upon $m$

$$
\mu=\alpha_{L}\left(1+2.5 \alpha_{G}\right) \mu_{L}+\alpha_{G} \mu_{G}
$$

In this work the RNG $k-\varepsilon$ turbulence model ${ }^{(16)}$ for high Reynolds number flow is adopted to take account of the turbulence effect and the Reynolds stress is given as follows.

$$
-\overline{\rho u_{i}^{\prime} u_{j}^{\prime}}=2 \mu_{t} S_{i j}-\frac{2}{3}\left(\mu_{t} \nabla \cdot \boldsymbol{u}+\rho k\right) \delta_{i j}
$$

where $\mu_{t}$ denotes the eddy viscosity which is defined by the following formula according to the turbulence energy $k$ and the turbulence dissipation rate $\varepsilon$.

$$
\mu_{t}=\chi \rho C_{\mu} k^{2} / \varepsilon
$$

in which $\chi$ is an eddy viscosity modification coefficient given as follows.

$$
\chi\left(\alpha_{G}\right)=\frac{1+\left(c_{\rho}-1\right)\left(1-\alpha_{G}\right)^{m}}{\alpha_{G}+c_{\rho}\left(1-\alpha_{G}\right)}
$$

where $c_{\rho}=\rho_{L} / \rho_{G}$, denotes the ratio of liquid and gas density and $m$ is an empirical parameter. Figure 1 shows the variation of modification coefficient $\chi$ when $m$ takes different values from 1 to 10 . As shown in the figure the value of $\chi$ is smaller than 0.2 when $\alpha_{G}$ is greater than 0.5 and $m$ is greater than 4. Cavitation bubbles included in liquid expand a/o contract with flow variation and the turbulence energy of bubbly flow may be absorbed. So, the eddy viscosity in cavitating region becomes much weak compared to the liquid flow region. Under this consideration, Kunz et al. treated bubbly flows by applying a high Reynolds number $k-\varepsilon$ model to the liquid flow field only ${ }^{(9)}$ and some cavitating flow simulations (11), (12) were carried without application of turbulence model although the Reynolds number of flows treated reaches to $3 \times 10^{5}$. To take account of the turbulence effect in high Reynolds number cavitating flows the RNG $k-\varepsilon$ model is adopted here and a modification of eddy viscosity is introduced by taking that $m=4$ according to the result shown in Fig.1.

Transportation equations of the turbulence energy and the turbulence dissipation rate are given as follows ${ }^{(17),(18)}$ considering the effect of cavitation.

$$
\begin{aligned}
\frac{\partial}{\partial t}(\rho k)+\nabla \cdot\left(\rho \boldsymbol{u} k-\left(\mu+\frac{\mu_{t}}{\sigma_{k}}\right) \nabla k\right)= & \mu_{t}\left(P+P_{B}\right)-\rho \varepsilon-\frac{2}{3}\left(\mu_{t} \nabla \cdot \boldsymbol{u}+\rho k\right) \nabla \cdot \boldsymbol{u} \\
\frac{\partial}{\partial t}(\rho \varepsilon)+\nabla \cdot\left(\rho \boldsymbol{u} \varepsilon-\left(\mu+\frac{\mu_{t}}{\sigma_{\varepsilon}}\right) \nabla \varepsilon\right)= & C_{\varepsilon 1} \frac{\varepsilon}{k}\left[\mu_{t} P-\frac{2}{3}\left(\mu_{t} \nabla \cdot \boldsymbol{u}+\rho k\right) \nabla \cdot \boldsymbol{u}\right] \\
& -C_{\varepsilon 2} \rho \frac{\varepsilon^{2}}{k}+C_{\varepsilon 3} \frac{\varepsilon}{k} \mu_{t} P_{B}+C_{\varepsilon 4} \rho \varepsilon \nabla \cdot \boldsymbol{u}
\end{aligned}
$$

where $P=2 s_{i j} \cdot \nabla \boldsymbol{u}, \quad P_{B}=\frac{1}{\rho \sigma_{h}} \boldsymbol{g} \cdot \nabla \rho, C_{\varepsilon 2} \equiv C_{\varepsilon 2}^{\sim}+\frac{C_{\mu} \lambda^{3}\left(1-\lambda / \lambda_{0}\right)}{1+\beta \lambda^{3}}, \quad \lambda \equiv \frac{k}{\varepsilon} \sqrt{2 s_{i j} s_{i j}}$ 
Empirical coefficients included in the above turbulence model are assigned as follows. $C_{\mu}=0.085, \sigma_{k}=0.719, \sigma_{\varepsilon}=0.719, \sigma_{h}=0.9, C_{\varepsilon 1}=1.42, C_{\varepsilon 2}^{\sim}=1.68, C_{\varepsilon 3}=1.42$, $C_{\varepsilon 4}=-0.387, \gamma_{0}=4.38, \quad \beta=0.012$.

In order to close above equations, Eq. (7) relating the mixture density and liquid pressure is taken into Eq. (9) and the following pressure transport equation is derived.

$$
\frac{\partial p}{\partial t}+\boldsymbol{u} \cdot \nabla p=-\rho c^{2} \nabla \cdot \boldsymbol{u}
$$

Furthermore, to estimate the variation of gas volume fraction the following gas mass conversation equation is employed.

$$
\frac{\partial}{\partial t}\left(\rho_{G} \alpha_{G}\right)+\boldsymbol{u} \cdot \nabla\left(\rho_{G} \alpha_{G}\right)=-\left(\rho_{G} \alpha_{G}\right) \nabla \cdot \boldsymbol{u}
$$

where $\rho_{G} \alpha_{G}$ denotes the gas mass fraction. Here the vapor evaporation and condensation in cavitation bubbles are supposed to be negligible for the purpose of simplification.

\subsection{Computational Algorithm}

Equations (9), (10), (16), (17), (18) and (19) compose a set of turbulent cavitating flow governing equations. Considering the coexistence of strong compressible bubbly flow region and weak compressible liquid flow region in cavitating flow field these equations are solved by the CIP-CUP method ${ }^{(19)}$ based on the time splitting technique ${ }^{(20)}$. The advection phase is calculated by CIP scheme, and the non-advection phase is calculated by finite difference method, where the viscous term and the acoustic term are respectively discretized by explicit and implicit schemes respectively. The mean pressure is calculated by solving the Poisson equation of pressure variation caused by flow compressibility ${ }^{(21)}$. Validations of the computer code may refer to reference (22).

\section{Computational Results}

\subsection{Water jet issuing from a sharp-edged orifice}

In order to confirm the validity of present method, submerged water jet issuing from a sharp-edged circular orifice is computed with $2 \mathrm{D}$ axi-symmetric assumption under different cavitation conditions and computational results are compared with experimental data ${ }^{(23)}$. Similar to Nurick's experiment ${ }^{(24)}$, geometry parameters of the orifice are given to be that $D / d=2.88$, and $L / d=5.0$, where $D, d$, and $L$ denote the inlet diameter, the orifice throat diameter, and the orifice length, respectively. As an index of cavitation condition, cavitation number $\sigma$ for the jet nozzle is defined as follows.

$$
\sigma=\frac{p_{o}-p_{v}}{P_{i n}-p_{o}}
$$

where $P_{\text {in }}$ denotes the total pressure at the inlet, $p_{o}$ the static pressure at the outlet and $p_{v}$ the saturated vapor pressure. Concerning the performance of jet nozzle, its discharge coefficient is then defined by the following formula.

$$
c_{d}=\frac{q_{m}}{0.25 \pi d^{2} \sqrt{2 \rho_{L}\left(P_{i n}-p_{o}\right)}}
$$

where $q_{m}$ denotes the mass flow rate trough the nozzle under given condition.

Figure 2 (a) shows, as a sample, the distribution of gas volume fraction in the cavitating jet when $\sigma=0$. The result demonstrates that cavitation bubbles concentrate in the shear layer around jet flow and the value gas volume fraction reaches to 0.5 locally. Under the effect of cavitation bubble the flow discharge coefficient varies with the cavitation number. Figure 2 (b) shows the value of discharge coefficient evaluated under different cavitation conditions, 


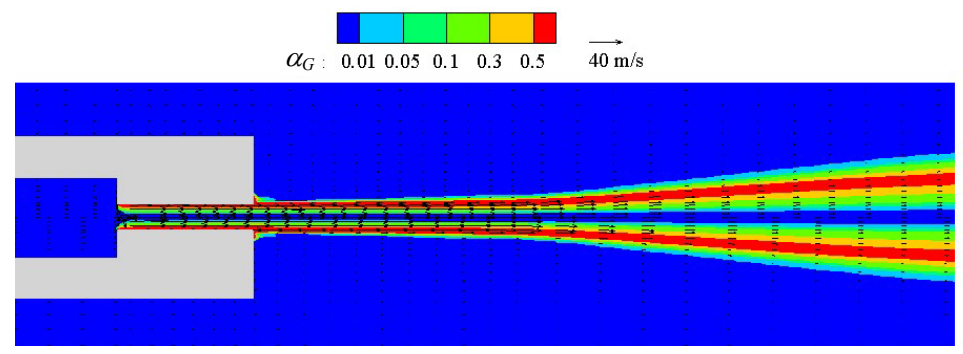

(a) Contour map of $\alpha_{G}$ when $\sigma=0.1$

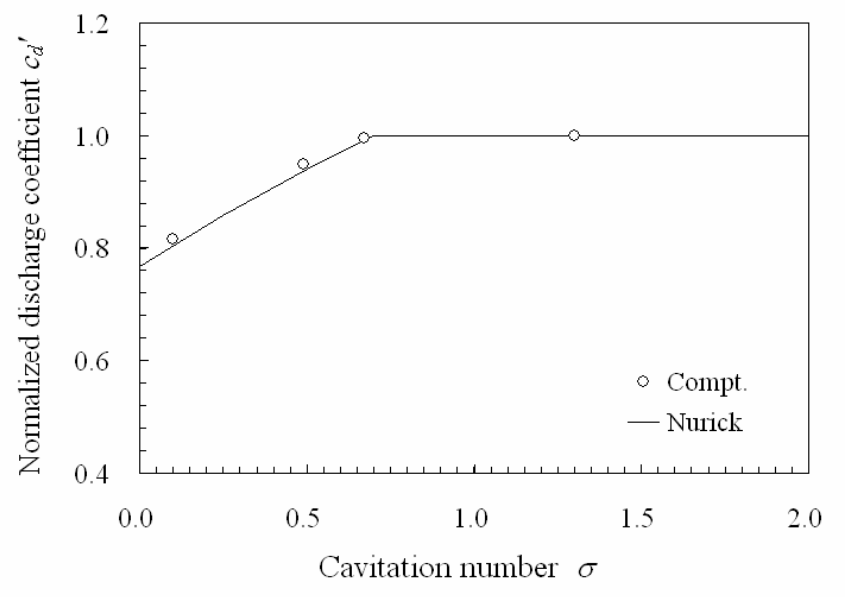

(b) Variation of discharge coefficient

Fig. 2 Evaluation of cavitating jet issuing from an orifice nozzle

where the vertical axis is normalized by the discharge coefficient of no-cavitating jet. The circles indicate computational results obtained by present method and the solid line refers to Nurick's experimental data ${ }^{(24)}$. The figure demonstrates that the computational results agree to the experimental data acceptably. It is confirmed that under the effect of cavitation bubbles the discharge coefficient of jet nozzle decreases compared to the case of no-cavitating flow.

\subsection{Water jet issuing from a convergent-divergent nozzle}

Pressure-driven flow issuing from a submerged Venturi type nozzle is often encountered in engineering practice and it has received much attention for the effect of cavitation. Its simulation is a challenging because the pressure difference driving a flow through a small nozzle can be very high, which may cause a strong cavitation. A convergent-divergent nozzle is adopted in this work and its geometry parameters are given as follows: $D_{1} / d=1.36, L_{1} / d=6.5 D_{2} / d=1.64$ and $L_{2} / d=8.5$, where $d$ denotes the diameter at the throat, $D_{1}$ does the diameters at the inlet of convergent section and $D_{2}$ does the diameters at the outlet of divergent section, $L_{1}$ and $L_{2}$ do the length of convergent and divergent sections from the throat to the inlet and the outlet, respectively.

Figure 3 shows the geometry of nozzle and the computation domain taken for flow

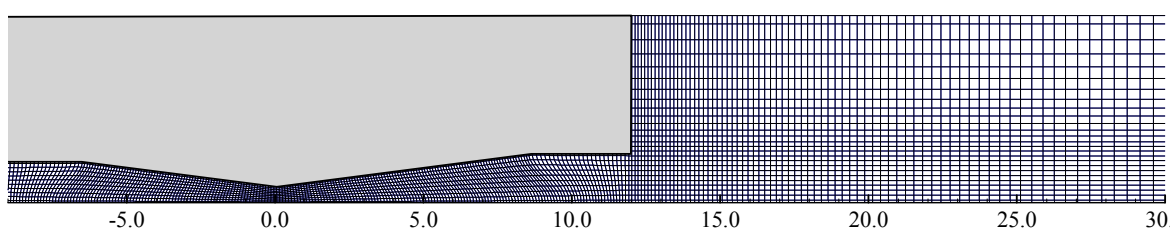

Fig. 3 Geometry of nozzle and computational domain adopted 
simulation. Focused on the axisymmetric vortex structure near the nozzle exit the flow is simplified into two-dimensional axisymmetric one and the computational domain is discretized with body-fitted structured grid as shown in the figure. The inlet of computation domain is taken to the $9.0 \mathrm{~d}$ upstream and the outlet is taken to $32.0 \mathrm{~d}$ downstream. Concern the boundary conditions of flow simulation, a uniform velocity condition is imposed at the inlet according to the given total pressure and corresponding flow velocity. The velocity turbulence is given to be $2 \%$ of the inlet velocity and then the values of kinetic energy and turbulence dissipation rate are determined ${ }^{(25)}$. At the outlet a given pressure is imposed and the normal gradient of other flow variables such as $u, k$ is set to be zero. The liquid boundary at the outside cylindrical surface is set to be free flow one. At the wall boundaries of nozzle geometry the no-slip condition of viscous fluid is imposed by applying the logarithmic wall function in the near region of wall ${ }^{(17)}$. By this way the need to employ fine computational cells within wall boundary layers, which is associated with computing cost, was avoided. At the axisymmetric axis of $r=1.0$ the Neumann condition $\partial / \partial r=0$ is applied to all flow variables.

Concerning the structure of no-cavitating jet, flow simulation is performed by specifying a discharge pressure high enough. Figure 4 shows, as an example, the distribution of no-cavitaing flow when the driven pressure $\Delta p \cong 1.0 \mathrm{MPa}(\sigma \geq 2.5)$. According to the computational results the mean velocity at the throat reaches to $42 \mathrm{~m} / \mathrm{s}$ and the Reynolds number is calculated to be $R e \cong 4.5 \times 10^{5}$. Figure 4 (a), (b), (c) and (d) respectively show the distributions of axial velocity, turbulence kinetic energy, turbulence dissipation rate, and pressure in dimensionless manner when the flow is well-developed, in which the solid black lines denote the distribution of temporal streamlines. As shown in the figures, the jet flow accelerates in the convergent section and the flow velocity reaches the maximum near the throat. In the divergent section a strong shear layer is formed and a small flow separation is demonstrated near the wall just behind the throat. According to Fig. 4 (b) and (c), we understand that the flow turbulence is caused mainly in the shear layer and the

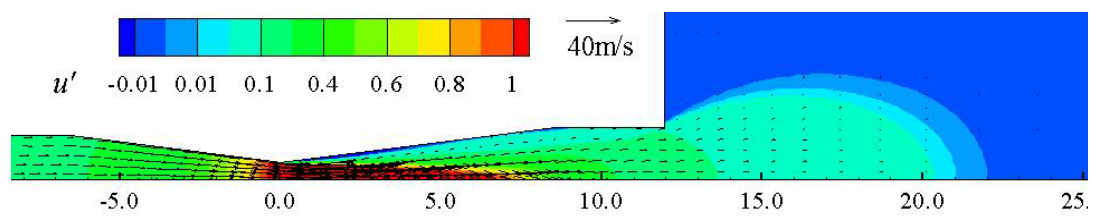

(a) Contour map of axial velocity $u / u_{0}$

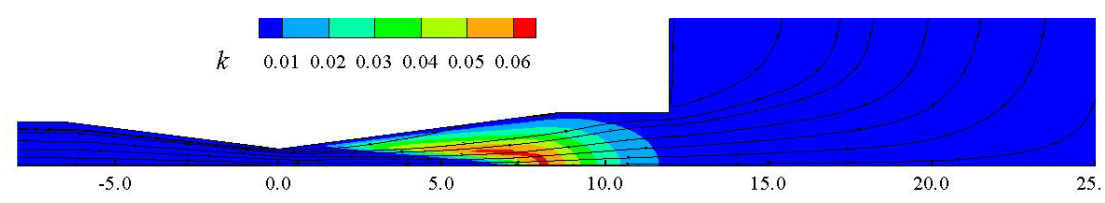

(b) Contour map of $k$

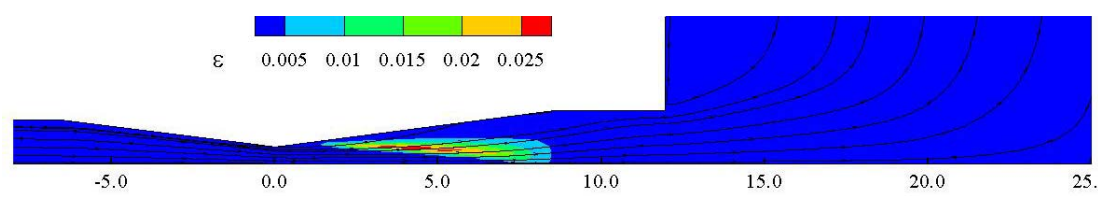

(c) Contour map of $\varepsilon$

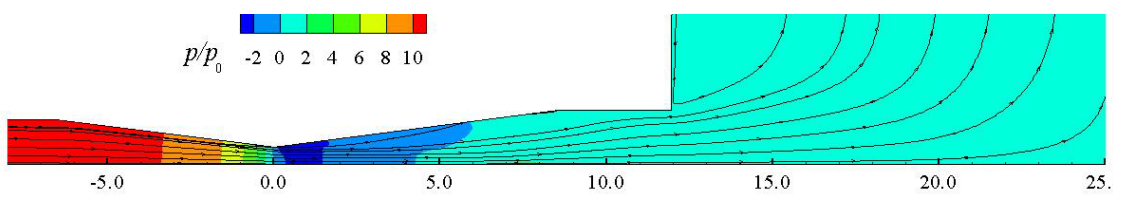

(d) Isobars

Fig. 4 Distribution of developed no-cavitating flow 
turbulence dissipation concentrates on the jet periphery. The pressure decreases from the inlet to the throat corresponding to the increase of flow velocity and a low-pressure region is formed just behind the throat as shown in Fig. 4 (d), where cavitation is easy to take place under a small cavitation number.

With a special concern on cavitation, jet flow issued at a small cavitation number are investigated. In this work the working liquid is taken to be general city water at $293 \mathrm{~K}$ of which the concentration of dissolved gas is $15 \mathrm{ppm}$ approximately. The dissolved gas is supposed to disperse in water in the form of bubble nuclear whose radius is given to be $10 \times 10^{-6} \mathrm{~m}$. The volume fraction of dissolved gas is set to be $1.0 \%$ at the initial state. Figure

(a)

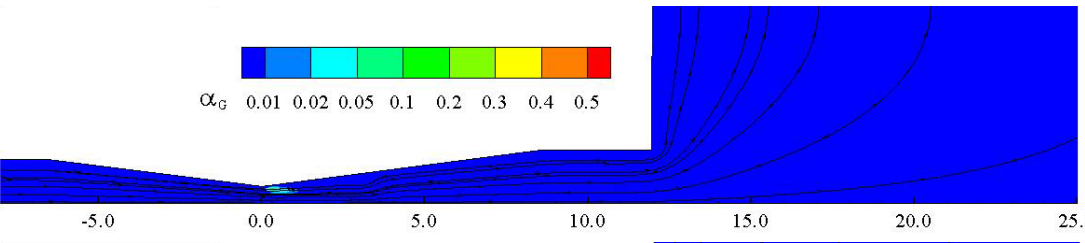

(b)

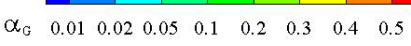

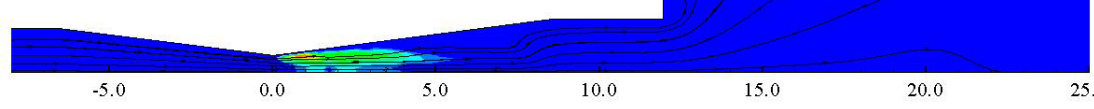

(c)

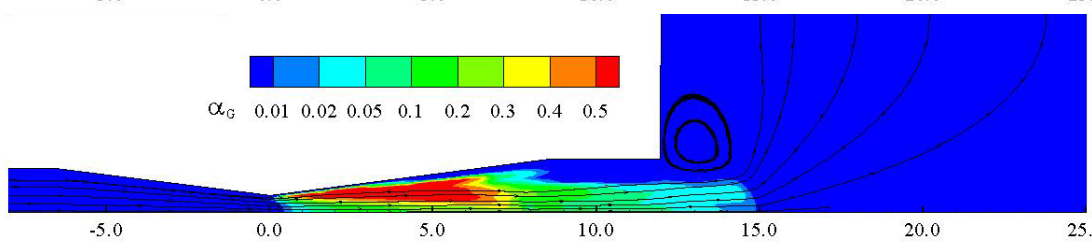

(d)

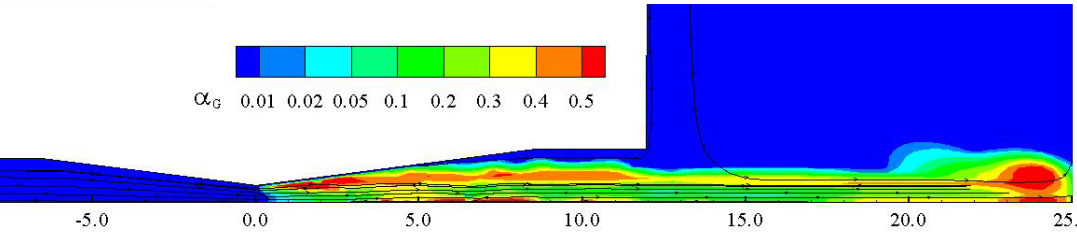

(e)

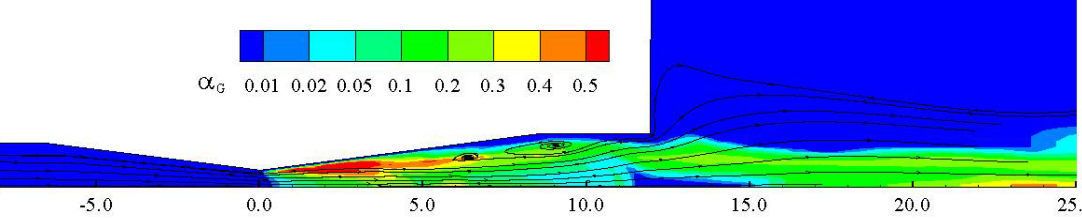

Fig. 5 Development of cavitaing jet $(\sigma=0.1)$

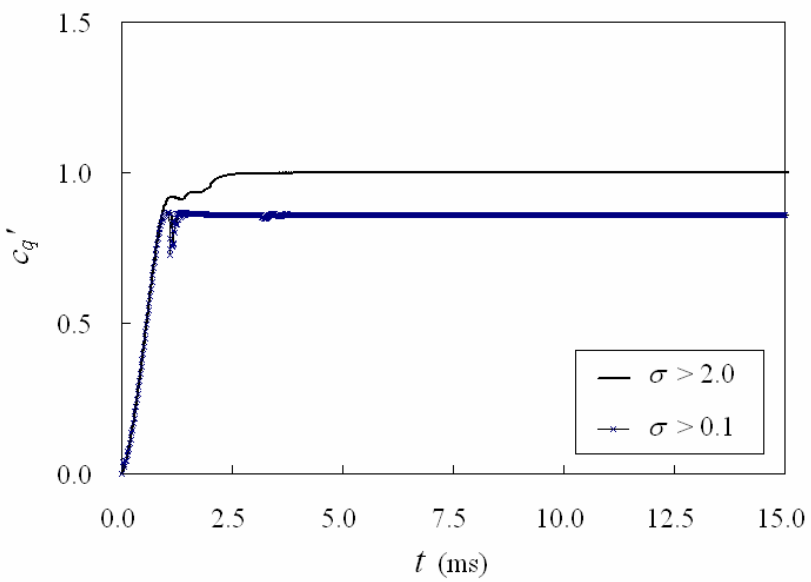

Fig. 6 Temporal variation of discharge coefficient 
5 shows, as a sample, the temporal variation of gas volume fraction when the driven pressure is kept to be $\Delta p \cong 1.0 \mathrm{MPa}$ but the cavitation number is decreased to 0.1 by adjusting the discharge pressure. Figure 5 (a) to (e) show the variation of gas volume fraction in a sequence time, where $\alpha_{G}$ increases to 0.5 locally with the development of jet flow. The result demonstrates that cavitation takes place initially in the low pressure area just behind the throat and the gas volume fraction $\alpha_{G}$ increases gradually with developing of jet. From Fig. 5 (b) and (c) we understand that cavitatition bubbles relatively concentrate in the shear layer near the throat in the divergent section. As shown in Fig. 5 (d) bubbles seem to be gradually transported into the starting vortex formed at the head of jet and a ring of cavitation bubbles appear within the starting vortex. Figure 5 (e) shows the flow structure at a well-developed stage where small shear vortexes are generated periodically in the shear layer of the divergent section. Cavitation takes place bear the wall behind the throat and cavitation bubbles flow downstream along the shear layer around jet periphery.

Figure 6 shows the temporal variation of discharge coefficient in the case of cavitating jet when $\sigma=0.1$ by the solid line with ${ }^{*}$ symbol. For comparison the thin solid line presents the variation of discharge coefficient in the case of no-cavitating jet. At the starting stage the driven pressure of water jet increases almost linearly and thus the flow discharge coefficient increases gradually to its maximum and then accommodates to a certain level. The result confirms that the discharge coefficient of present convergent-divergent nozzle decreases obviously under the influence of cavitation. Under the given condition that $\sigma=$ 0.1 , the discharge coefficient decreases to $85 \%$ approximately compared to the case of no-cavitating flow. However, the maximum jet velocity in the throat increases inversely in a certain extent and the decline of jet velocity becomes slower compared to the case of pure water jet for the effect of bubble expansion and contraction.

\section{Conclusions}

A practical compressible mixture flow method is developed for the computation of high Reynolds number turbulent cavitating flow by coupling a simplified estimation of bubble cavitation to the compressible mixture flow computation procedure based on the CIP-CUP method. The method has been applied to high-speed submerged water jets accompanied with intensive cavitation and its reliability is confirmed.

Concerning the structure of water jet issuing from a submerged convergent-divergent nozzle, computational results demonstrate that pressure decreases with flow acceleration in the convergent section and a low pressure region is formed just behind the nozzle throat. Cavitation occurs initially in the low-pressure region between the wall and the shear layer just behind the throat. When the cavitation number is decreased to 0.1 the gas volume fraction of cavitation bubbles increases in a sequence time and its maximum value reaches to 0.5 locally. As a jet gets to be well developed cavitation takes place continually in the shear layer behind the nozzle throat and bubbles flow downstream along the jet periphery mainly. Under the effect of cavitation bubbles the mass discharge coefficient decreases obviously compared to the case of no-cavitating jet although the maximum jet velocity in the throat increases inversely in a certain extent.

\section{Acknowledgements}

This work was supported by Grant-in-Aid for Scientific Research (C) (No. 22560177).

\section{References}

(1) Soyama, H. et al. High-speed observation of ultrahigh-speed submerged water jets, Experimental Thermal and Fluid Science, Vol.12 (1996), pp.411-416. 
(2) Foldyna, J., Sitek, L., Svehla, B. and Svehla, S., Utilization of ultrasound to enhance high-speed water jet effects, Ultrasonics Sonochemistry, Vol.11 (2004), pp.131-137.

(3) Gopalan, G., Katz, J. and Knio, O., The flow structure in the near field of jets and its effect on cavitation inception, J. Fluid Mech. Vol.398 (1999), pp.1-43.

(4) Ventikos, Y. and Tzabiras, G., A numerical method for the simulation of steady and unsteady cavitating flows, Computers \& Fluids, Vol.29 (2000), pp.63-88.

(5) Wang G. et al., Dynamics of attached turbulent cavitating flows, Progress in Aerospace Science, Vol.37 (2001), pp.551-581.

(6) Sussman, M., A second order coupled level set and volume-of-fluid method for computing growth and collapse of vapor bubbles, J. Computational Physics, Vol.187 (2003), pp.110-136.

(7) Egashira, R., Yano, T. and Fujikawa, S., Linear wave propagation of fast and slow modes in mixtures of liquid and gas bubbles, Fluid Dynamic Research, Vol.34 (2004), pp.317-334.

(8) Delale, C. F, Okita, K., and Matsumoto, Y., Steady-state cavitating nozzle flows with nucleation, J. Fluids Eng., Vol.127 (2005), pp.770-777.

(9) Kunz, R. T. et al., Validation of two-fluid Eulerian CFD modeling for microbble drag reduction across a wide range of Reynolds numbers, J. Fluids Eng., Vol.129 (2007), pp.66-79.

(10) Singhal, A. K. et al., Mathematical basis and validation of the full cavitation model, $J$. Fluids Eng., Vol.124 (2002), pp.617-624.

(11) Kubota, A., Kato, H. and Yamaguti, H., A new modeling of cavitating flow: A numerical study of unsteady cavitation on a hydrofoil section, J. Fluid Mech., Vol.240 (1992), pp.59-96.

(12) Iga, Y. et al., Numerical study of sheet cavitation breakoff phenomenon on a cascade hydrofoil, J. Fluids Eng., Vol.125 (2003), pp.643-651.

(13) Peng, G., Fujikawa, S., Ishizuka, M. and Hayama, S., Numerical simulation of submerged water jet by an improved CIP-CUP method, Computational Fluid Dynamics $J .$, Vol.11 (2002), pp.27-34.

(14) Peng, G., et al., A compressible two-phase flow bubble cavitation model for computation of cavitating flows, Proc. 1st Int. Colloquium on Dynamics, Physics and Chemistry of Bubble and Gas-Liquid Boundaries (ICBB2007), Hokkaido, Sept. 25-28, 2007, Paper No. 2512 (CD-ROM).

(15) Beattie, D. and Whally, P., A simple two-phase frictional pressure drop calculation method, Int. J. Multiphase Flow, Vol.8 (1982), pp.83-87.

(16) Yakhot, V. et al. Development of turbulence models for shear flows by a double expansion technique. Phys. Fluids, A4, No.7 (1992), pp.1510-1520.

(17) Wilcox, D. C., Turbulence modeling for CFD, 2nd ed. DCW Industries, 2002.

(18) Peng, G., Shimizu S. and Fujikawa, S., A numerical study of turbulent vortex cavitating flows in a submerged water jet, J. Jet Flow Eng., Vol. 25, No.2 (2008), pp.11-17. (in Japanese)

(19) Yabe, T. and Aoki, T., A universal solver for hyperbolic equations by cubic-polynomial interpolation, Computer Physics Communications, Vol.66, (1991), 219-242.

(20) Issa, R. I., Solution of the implicitly discretised fluid flow equations by operatorsplitting, J. Comp. Phys. Vol.62 (1986), pp.40-65.

(21) Peng, G., Egashira, R., Yano, T. and Fujikawa, S., A pressure-based two-phase flow method for computation of bubble cavitation flows, Proc. 5th Joint ASME/JSME Fluids Eng. Conf., San Diego, USA, July 30-Aug.2, 2007, Paper No. FEDSM2007-37420.

(22) Peng, G., Ishizuka, M. and Hayama, S., An improved CIP-CUP method for submerged water jet flow simulation, JSME Int. J. (B): Fluids and Thermal Eng., Vol.44, No.4 (2001), pp.497-504. 
(23) Peng, G., Shimizu, S. and Fujikawa, S., Numerical simulation of turbulent cavitating water jets issued from a submerged orifice nozzle, Proc. 9th Prim Rig Int. Conf. on Water Jets, Koriyama, Japan, Nov. 19-23, 2009, pp.139-144.

(24) Nurick, W. H., Orifice cavitation and its effect on spray mixing, J. Fluids Eng., Vol.98 (1976), pp.681-687.

(25) Cao, S., Peng G. and Yu Z., Hydrodynamic design of rotodynamic pump impeller for multiphase pumping by combined approach of inverse design method and CFD analysis, J. Fluids Eng., Vol. 127 (2005), pp.330-338. 